\title{
The pronounced high expression of discoidin domain receptor 2 in human interstitial lung diseases
}

\author{
Huan Bian ${ }^{1,2,5}$, Xiaowei $\mathrm{Nie}^{3,5}$, Xin $\mathrm{Bu}^{2,5}$, Feng Tian ${ }^{4}$, Libo Yao ${ }^{2}$, Jingyu Chen ${ }^{3}$ \\ and Jin $\mathrm{Su}^{1,2}$
}

Affiliations: 'State Key Laboratory of Respiratory Diseases, Guangzhou Institute of Respiratory Diseases, The First Affiliated Hospital of Guangzhou Medical University, Guangzhou, PR China. ${ }^{2}$ State Key Laboratory of Cancer Biology, Dept of Biochemistry and Molecular Biology, The Fourth Military Medical University, Xi'an, China. ${ }^{3}$ Jiangsu Key Laboratory of Organ Transplantation, Wuxi People's Hospital, Nanjing Medical University, Wuxi, PR China. ${ }^{4}$ Dept of Thoracic Surgery, Tangdu Hospital, The Fourth Military Medical University, Xi'an, China. ${ }^{5}$ These authors contributed equally.

Correspondence: Jin Su, 151 Yanjiang Road, Guangzhou, 510120, China. E-mail: sujindagird.cn

ABSTRACT The most typical structural feature of human interstitial lung diseases (ILDs) is the accumulation of vast amounts of collagens within the lung interstitium. The membrane receptors that are responsible for recognising collagens and then transducing signals into the cells include four members of the integrin family $(\alpha 1 \beta 1, \alpha 2 \beta 1, \alpha 10 \beta 1$ and $\alpha 11 \beta 1)$ and two members of the discoidin domain receptor family (DDR1 and DDR2). However, it remains unknown whether these six collagen receptors similarly contribute to the pathogenesis of fibrotic lung diseases.

Quantitative real-time PCR (qPCR) was utilised to assess the mRNA expression of the genes studied. Immunoblot experiments were performed to analyse the protein abundance and kinase activity of the gene products. The tissue location was determined by immunohistochemical staining.

qPCR data showed that DDR2 mRNA displays the most dramatic difference between idiopathic pulmonary fibrosis (IPF) patients and healthy groups. The outstanding increases in DDR2 proteins were also observed in some other types of ILD besides IPF. DDR2-expressing cells in ILD tissue sections were found to exhibit spindle or fibroblastic shapes.

Our investigation suggests that DDR2 might represent a major cell surface protein that mediates collagen-induced cellular effects in human ILD and, hence, is suitable for their diagnosis and therapy.

@ERSpublications

DDR2 is a major collagen-recognising receptor in human interstitial lung disease http://ow.ly/Lhgh30gN603

Cite this article as: Bian $\mathrm{H}, \mathrm{Nie} \mathrm{X}, \mathrm{Bu} \mathrm{X}$, et al. The pronounced high expression of discoidin domain receptor 2 in human interstitial lung diseases. ERJ Open Res 2018; 4: 00138-2016 [https://doi.org/ 10.1183/23120541.00138-2016].

This article has supplementary material available from openres.ersjournals.com

Received: Nov 282016 | Accepted after revision: Oct 292017

Support statement: This work was supported by the National Natural Science Foundation of China (31671472 and 81500039), the Science and Technology Agency of Jiangsu Province (BM2012108), and the Chinese National Key Basic Research and Development Program (2010CB529705). Funding information for this article has been deposited with the Crossref Funder Registry.

Conflict of interest: None declared.

Copyright $\odot$ ERS 2018. This article is open access and distributed under the terms of the Creative Commons Attribution Non-Commercial Licence 4.0. 


\section{Introduction}

Pulmonary fibrosis, characterised by progressive and irreversible scar formation in the lung interstitium and by the gradual reduction in alveolar space, is the final stage of most interstitial lung diseases (ILDs) and can eventually lead to malfunction of gas exchange and subsequent respiratory failure. Its known causes (risk factors) include certain environmental exposures or insults, such as cigarette smoking and microaspiration [1]. Pulmonary fibrosis also is the common complication of some connective tissue diseases, with systemic sclerosis (scleroderma) and rheumatoid arthritis as the two prominent examples. When all known causes of ILD have been ruled out, this condition is called idiopathic pulmonary fibrosis (IPF). IPF represents the most prevalent and lethal form of ILD, and patients usually have a median survival of only $2-5$ years after diagnosis $[2,3]$. The 5 -year survival rates in IPF are even lower than in the most types of aggressive cancer [4]. Despite that two pharmacological agents have recently entered clinical use for the treatment of IPF on the basis of their efficacy in reducing the decline in pulmonary function, huge challenges remain because these agents failed to improve mortality outcomes [5-7].

It has been generally accepted that some soluble cytokines, like transforming growth factor (TGF)- $\beta$ and connective tissue growth factor, play active roles in the initiation of dysregulated fibrotic reactions in the lung $[8,9]$, with strategies targeting these molecules or related pathways being under clinical evaluation $[10,11]$. Nevertheless, the fact is that IPF is diagnosed relatively late and the lung environment of such patients receiving management may have become dramatically different from that during initiation or early progression [12]. Several recent studies and reviews have suggested the concept that the excessively deposited extracellular matrix (ECM) proteins in the fibrotic lung may be drivers of, rather than bystanders in, the progression of pulmonary fibrosis [13-16]. Considering that collagens are major, as well as the most abundant, components of ECM, it is highly likely that those cell surface receptors responsible for transducing signals from collagens into the cells might be important intervention targets.

In mammals, there are primarily two diverse types of collagen receptor, the integrin and discoidin domain receptor (DDR) families [17, 18]. The DDR family, consisting of DDR1 and DDR2, are receptor tyrosine kinases and their intracellular domains can be induced into a sustained tyrosine-phosphorylated state in response to collagen binding, which ultimately triggers an array of signalling pathways and cellular effects $[19,20]$. Recently, it was suggested that DDRs are also able to elicit influence on cells in a ligand binding-independent manner [21, 22]. Integrins are totally different from DDRs in that they are heterodimers formed by noncovalent association of one $\alpha$ subunit (of which there are at least 18) and one $\beta$ subunit (of which there are at least eight), and do not have the kinase activity [23]. Among the 24 known $\alpha \beta$ pairs, there are four different collagen-binding integrins: $\alpha 1 \beta 1, \alpha 2 \beta 1, \alpha 10 \beta 1$ and $\alpha 11 \beta 1[17,24]$.

Currently, only four collagen receptors have been reported to be linked to the pathogenesis of lung fibrotic diseases: DDR1, DDR2, and $\alpha 2 \beta 1$ and $\alpha 11 \beta 1$ integrins. Compared to lung fibroblasts from normal individuals, those from IPF patients were found to express aberrantly low levels of $\alpha 2 \beta 1$ integrin, which were experimentally confirmed to result in a failure of integrin to induce protein phosphatase $2 \mathrm{~A}$ as well as the inappropriate activation of the $\beta$-catenin proliferation signal pathway [25]. As for the contribution of $\alpha 11 \beta 1$ integrin to pulmonary fibrosis, it was documented that the expression of CIB1, which is a binding partner of integrin $\alpha 11$ subunit (ITGA11), was elevated in mouse fibrotic lung tissues and its overexpression can suppress the activation of human lung fibroblast cell lines [26]. Data from our laboratory and others demonstrated that the mice deficient in either Ddr1 or Ddr2 are both resistant to bleomycin-induced experimental pulmonary fibrosis [22, 27]. The detailed mechanism underlying DDR1 regulation of this type of pathological disorder remains unclear, whereas DDR2 has been proven to control the activation of lung myofibroblasts by mediating the signalling triggered by collagen and TGF- $\beta$ [22].

Our preliminary findings derived from immunohistochemical examination of a very limited number of IPF samples hint that DDR2 expression in IPF lungs is much higher than that in healthy controls [22]. In view of the above research situation, several uncertainties remain. A first question is whether the expression signature of DDR2 in IPF can be reproduced in large quantities of clinical specimens or even in other types of ILD. The second question is that how is the expression abundance of the other five collagen receptor members in IPF. Another interesting and attractive issue may be whether there is one or several collagen receptors whose expression and function dominate in fibrotic lung diseases.

In the current study, we found that among the six members, the mRNA expression levels of only ITGA11, $D D R 1$ and $D D R 2$ in IPF patients dramatically exceed those in healthy controls, with this difference being most pronounced for DDR2.The sharp elevation of DDR2 expression in human fibrotic lung tissues could be further confirmed in the protein level. Therefore, our investigation suggests that DDR2 might represent a major collagen-recognising cell surface protein in human pulmonary fibrosis. 


\section{Methods}

Collection of human ILD specimens

All the ILD specimens were obtained from patients who had undergone lung transplantation surgery at the Key Laboratory of Organ Transplantation of Wuxi People's Hospital (Wuxi, PR China). The diagnosis of IPF was determined by each subject's primary pulmonologist according to American Thoracic Society/ European Respiratory Society guidelines incorporating high-resolution computed tomography characteristics, surgical lung biopsy data and clinical exclusion of alternative causes of ILD. In addition to IPF $(n=20)$, lung tissues of patients diagnosed with pulmonary fibrosis of other aetiologies (i.e. nonspecific interstitial pneumonia and connective tissue disease-associated ILD, etc.) were also included in this study $(n=14)$. The diagnostic information for these non-IPF patients was based on multidisciplinary approach and provided in the supplementary table. The 10 healthy lung tissue samples were from organ donors diagnosed with brain death. Written consent for tissue donation for research purposes was obtained from each patient or the family members of healthy donors before tissue collection. The study protocol was approved by the institutional review boards of Guangzhou Medical University (Guangzhou, PR China), the Fourth Military Medical University (Xi'an, PR China) and Wuxi People's Hospital.

\section{Preparation of total RNA and protein samples}

For extraction of RNA and protein simultaneously, the lung tissues were ground with TRIzol Reagent (Invitrogen, Carlsbad, CA, USA). The aqueous phase was used to extract RNA through isopropanol and ethanol precipitation steps. The organic phase was subjected to protein extraction according to the manufacturer's instruction. Briefly, after isopropanol and ethanol precipitation, the centrifuged pellets were washed sequentially three times with a solution containing $0.3 \mathrm{M}$ guanidine hydrochloride in $95 \%$ ethanol and once with pure ethanol. Finally, the obtained protein sediments were dissolved in protein sample buffer after vacuum drying.

\section{Quantitative real-time PCR}

cDNA was synthesised using the Super-Script II First-Stand Synthesis System (Invitrogen). Two-step quantitative real-time PCR (qPCR) with SYBR Green I (Takara, Shiga, Japan) labelling was conducted on a real-time thermocycler (Applied BioSystems, San Diego, CA, USA). The primer sequences used were as follows. Human ITGA1: GTCCAGTTGGGAGAGGTGAA (forward); CTCCATTTGGGTTGGTGACT (reverse); human ITGA2: GATTTGCGTGTGGACATCAG (forward); AAAGTCCGTCCTCACCACAG (reverse); human ITGA10: TCTTGGAGGTGGTTCAGACC (forward); AAAGAAGCCAAGCTTCCACA (reverse); human ITGA11: ACCCCATTTCCAAACAACAA (forward); CAGTACGGCTCTGTTGGTGA (reverse); human DDR1: GATTTCCCCCTTAATGTGCGT (forward); TGGCATCTGGCCGTAAGATC (reverse); human DDR2: CTCCCAGAATTTGCTCCAG (forward); GCCACATCTTTCCTGAGA (reverse); $\beta$-actin: TGGCACCCAGCACAATGAA (forward); TAAGTCATAGTCCGCCTAGAAGCA (reverse). The amplification programme was as follows: $95^{\circ} \mathrm{C}$ for $10 \mathrm{~s}$, followed by 40 cycles of $95^{\circ} \mathrm{C}$ for $5 \mathrm{~s}$ and $60^{\circ} \mathrm{C}$ for $34 \mathrm{~s}$. qPCR data were expressed as the mRNA expression level of the target gene relative to an internal reference control. Bar graphs represent the mean \pm SD of three individual experiments.

\section{Immunoblot analysis}

The protein lysates were resolved by $10 \%$ SDS-PAGE and then transferred to nitrocellulose membranes (Amersham Biosciences, Little Chalfont, UK). After blocking with 5\% bovine serum albumin in Tween 20-Tris-buffered saline, the membranes were incubated with primary antibodies, followed by incubation with horseradish peroxidase-labelled secondary antibodies (Cell Signaling Technology, Beverly, MA, USA). The protein bands were visualised using the ECL Western Blotting Detection Kit (GE Healthcare, Little Chalfont, UK). The primary antibodies used for immunoblot detection in this study were: DDR2 (1:1000; R\&D Systems, Minneapolis, MN, USA); collagen I (1:1000; Abcam, Cambridge, MA, USA); fibronectin (1:100; Sigma-Aldrich, St Louis, MO, USA); phosphorylated DDR2 (1:500; R\&D Systems); and tubulin (1:1000; Booster, Wuhan, PR China).

\section{Immunohistochemical staining}

For immunohistochemical detection of DDR2, paraffin sections $(4 \mu \mathrm{m})$ were dewaxed and rehydrated through graded alcohol, then subjected to DDR2 antigen retrieval by high pressure cooking for $2 \mathrm{~min}$ in $10 \mathrm{mmol} \cdot \mathrm{L}^{-1}$ citrate acid ( $\mathrm{pH}$ 6.0). The endogenous peroxidase activity was cleared using $3 \% \mathrm{H}_{2} \mathrm{O}_{2}$ in methanol for $10 \mathrm{~min}$. The sections were incubated with normal goat serum at a dilution of 1:100 for $1 \mathrm{~h}$. The antibody against human DDR2 was used at a dilution of 1:100. After incubation with the primary antibody or mouse IgG overnight at $4^{\circ} \mathrm{C}$, the sections were washed in PBS and incubated with biotinylated goat anti-mouse IgG at a dilution of 1:200 and VECTASTAIN ABC Reagent (Vectastain Elite ABC kit; Vector Laboratories, Burlingame, CA, USA) at a dilution of 1:400 consecutively for $30 \mathrm{~min}$ each. 
Peroxidase activity was demonstrated by adding 3,3'-diaminobenzidine as a chromogen (Sigma-Aldrich). The sections were then counterstained with haematoxylin, followed by examination under a light microscope. Negative controls were made by using normal mouse IgG on all tissue slides.

\section{Statistical analysis}

Statistical analyses were performed using the Statistical Product and Service Solutions software (IBM, Armonk, NY, USA). Independent-samples t-tests was used to examine the differences between the two groups of data. A two-tailed p-value of $<0.05$ was considered significant.

\section{Results}

mRNA expression profiles of six collagen receptors in IPF

Firstly, we conducted qPCR to compare the mRNA expression of the six collagen receptors between IPF and healthy donors. The expression levels of both ITGA1 and ITGA10 in IPF samples showed a slight decrease, whereas ITGA2 and ITGA11 were significantly upregulated, with mean fold changes of 2 and 5, respectively (figure 1). Unlike the mRNA of DDR1, the IPF-associated elevation of which was, on average, merely approximately seven-fold with large variations among different individual specimens (range from 1.4- to 23.3-fold), that of DDR2 reached up to 100-fold with little variation (range 59.3-191.8-fold) (figure 2a). The disease-induced expression enhancement of DDR2 also dramatically exceeded that of DDR1 in non-IPF ILD (figure $2 \mathrm{~b}$ ).

\section{Protein expression of DDR2 in ILD}

On the basis of the above data, we investigated the protein abundance of DDR2 by Western blotting. The results showed that the lung homogenates from $20 \mathrm{IPF}$ patients ubiquitously contained much more DDR2 protein as well as the fibrotic markers collagen I and fibronectin than those from 10 normal subjects (figure 3a). The expression level of DDR2 in non-IPF ILD cases also significantly exceeded that in the healthy group, albeit not as high as in IPF (figure 3b).

\section{Localisation and activation status of DDR2 in ILD}

Immunohistochemical experiments were further performed in order to locate the DDR2-expressing cells. Consistent to our recent findings in IPF [22], DDR2 was found to be highly expressed by the cells that display spindle or fibroblastic shapes in non-IPF ILD (figure 4).

The DDR2 proteins on the cell surface of these pathological fibroblasts theoretically have the chance to be triggered to activation by the abundantly accumulated fibrillar collagens within the fibrotic lesions that serve as its potent ligands [20]. In order to test this possibility, immunoblots were probed with a specific antibody against the tyrosine phosphorylated form of DDR2. The expected bands were more or less observable in both disease groups but were undetectable in healthy controls. Moreover, the phosphorylation level of DDR2 in IPF was much higher than that in non-IPF ILD (figure 5).

\section{Discussion}

Although four members of the family of collagen receptors (DDR1, DDR2, and $\alpha 2 \beta 1$ and $\alpha 11 \beta 1$ integrins) have been reported to be associated with the pathogenesis of lung fibrosis [22, 25, 27, 28], it remains unclear whether they and the other two members have similar abundance patterns or play equal roles in this type of disease. In this investigation, we revealed for the first time that, among the six collagen receptors, DDR2 is the only gene whose transcription and expression display sharp differences between IPF patients and healthy individuals. Considering that DDR2 plays a profibrotic role in experimental pulmonary fibrosis [22], our current data further provide clinical evidence that this unique collagen receptor can be viewed as a promising target for diagnosis and therapy of end-stage IPF.

The immunohistochemical findings from a previous publication showed that the expression level of ITGA1 in IPF fibroblastic foci was remarkably lower than that in the epithelial cells overlying the fibroblastic foci [25]. This result perfectly accounts for the tiny increase in ITGA2 observed in our study since qPCR experiments were conducted by use of whole-lung homogenates, which may contain both ITGA2-low expressing fibroblasts and ITGA2-high expressing epithelial cells. Due to the negative influence of ITGA2 signalling on IPF fibroblast proliferation [25], we speculate that it might not be an ideal therapeutic target for IPF.

According to the data here presented, ITGA11 appears to be the only collagen-binding integrin that is dramatically upregulated in all IPF samples used. This phenomenon seems to conform to the observations documented in two previous literature reports. One described that the expression of ITGA11 is enhanced in TGF- $\beta$-treated lung fibroblasts [28]. The other recorded that the level of an ITGA11 binding partner was elevated in mouse fibrotic lung tissues [26]. We also noticed that the fold increase in ITGA11 mRNA 

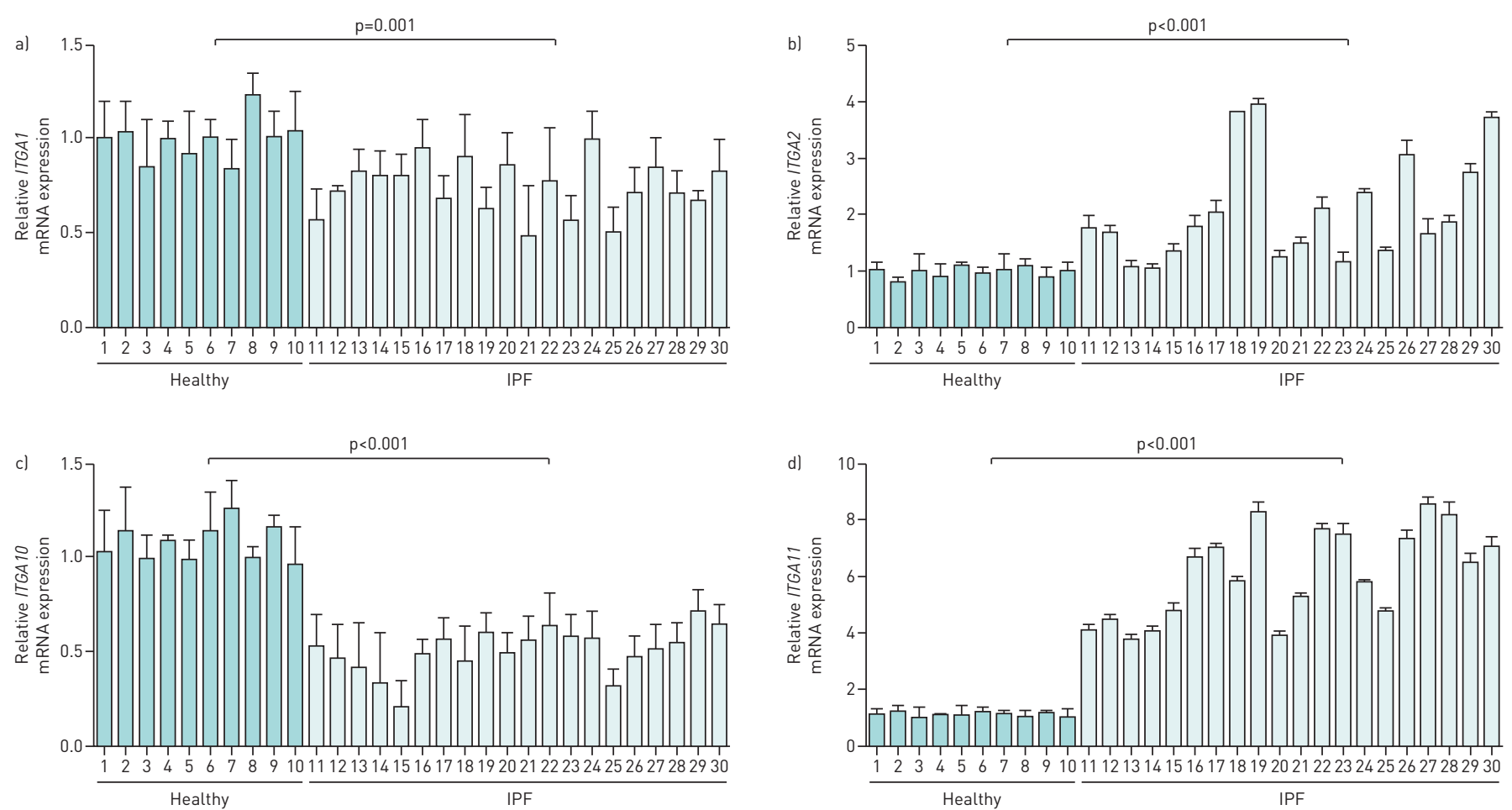

FIGURE 1 mRNA expression of collagen-binding integrins in idiopathic pulmonary fibrosis (IPF) lungs. The lung tissue cDNA from 20 IPF patients and 10 healthy donors were subjected to quantitative real-time PCR analysis of the mRNA expression levels of a) integrin $\alpha 1$ (ITGA1), b) ITGA2, c) ITGA10 and d) ITGA11, respectively. The graph indicates the expression level of the target gene relative to the reference gene $\beta$-actin. Bar graphs represent the mean \pm SD of three individual experiments.
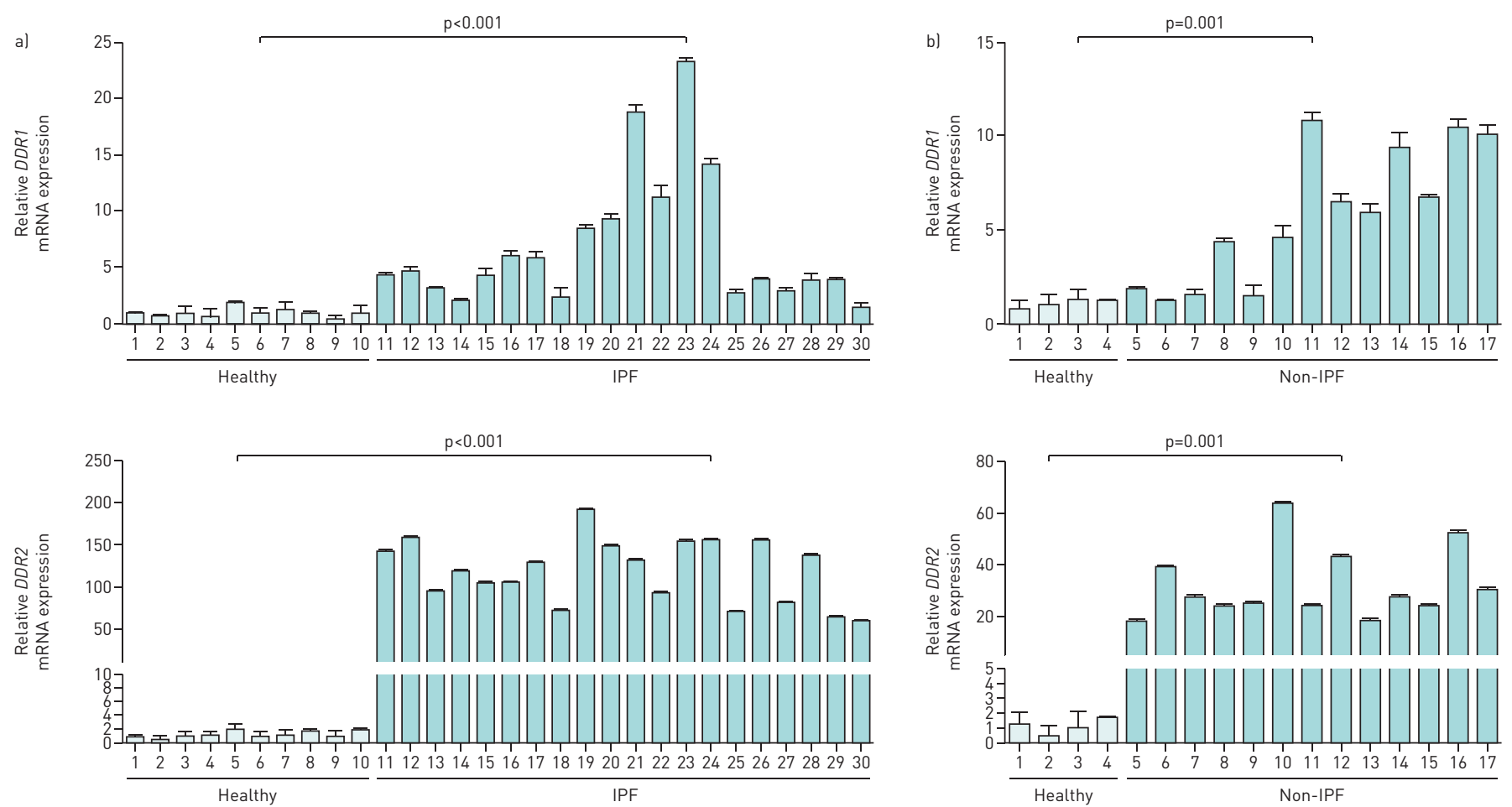

FIGURE 2 Comparative expression of discoidin domain receptors (DDRs) between healthy lung tissues and those from different types of interstitial lung disease (ILD): a) idiopathic pulmonary fibrosis (IPF) and b) non-IPF ILD. The disease-induced elevation of DDR2 dramatically exceeds that of $D D R 1$ in both IPF and non-IPF ILD. Bar graphs represent the mean \pm SD of three individual experiments. 
a)
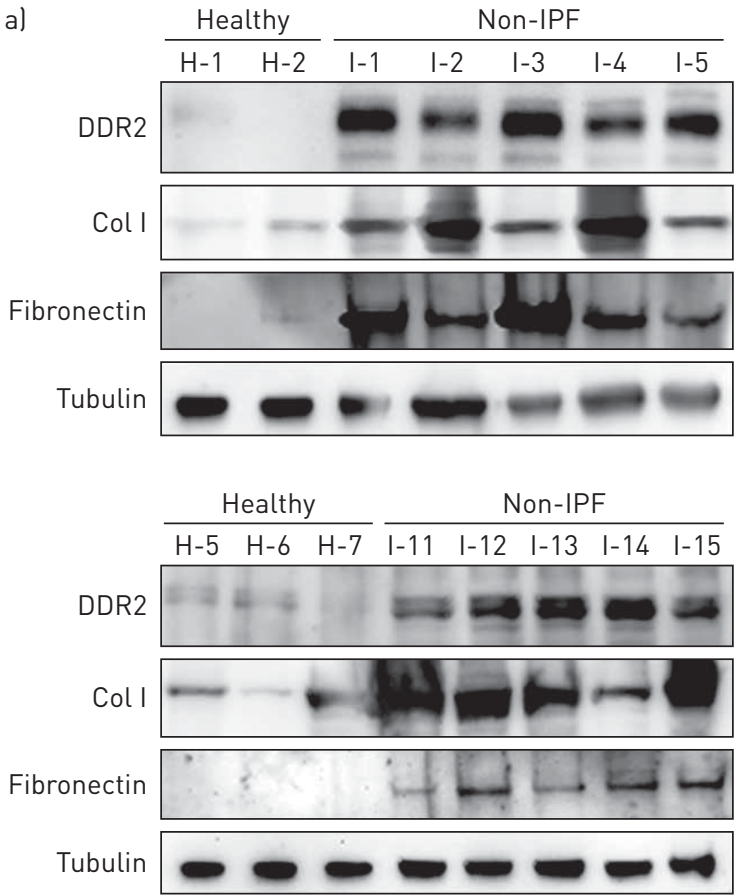

b)

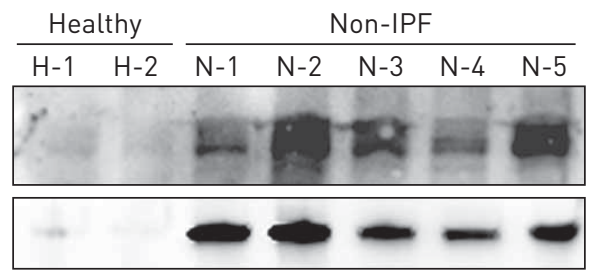

Fibronectin

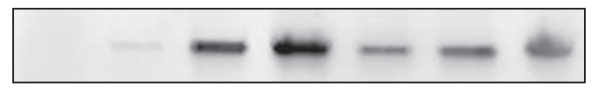

Tubulin

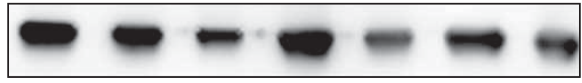

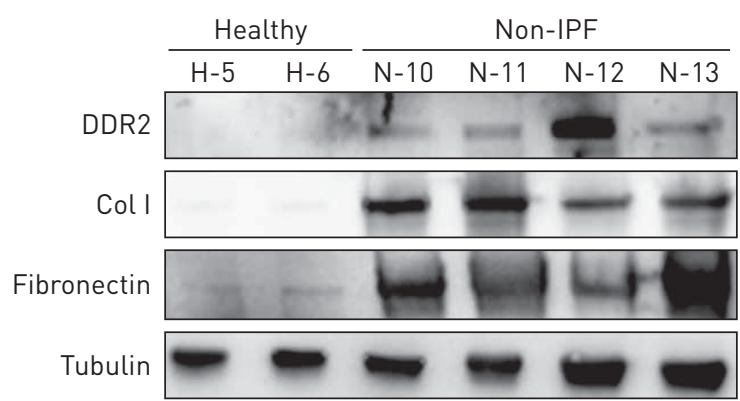

FIGURE 3 Discoidin domain receptor (DDR)2 protein was highly expressed in the lungs of patients with interstitial lung disease (ILD). The total lung proteins were extracted simultaneously with RNA by use of the standard TRIzol Reagent protocol and then subjected to immunoblot analysis of DDR2, collagen (Col) I, fibronectin and tubulin. DDR2 expression was greatly increased in both a) idiopathic pulmonary fibrosis (IPF) and b) non-IPF ILD.

expression in IPF versus controls was relatively close between patients. A possible explanation for this consistent upregulation may be that ITGA11 expression is predominantly restricted to fibroblasts [29]. As for the functional role of ITGA11 in lung fibroblasts, the only clue is the observation that its binding protein suppresses myofibroblast formation [26]. 

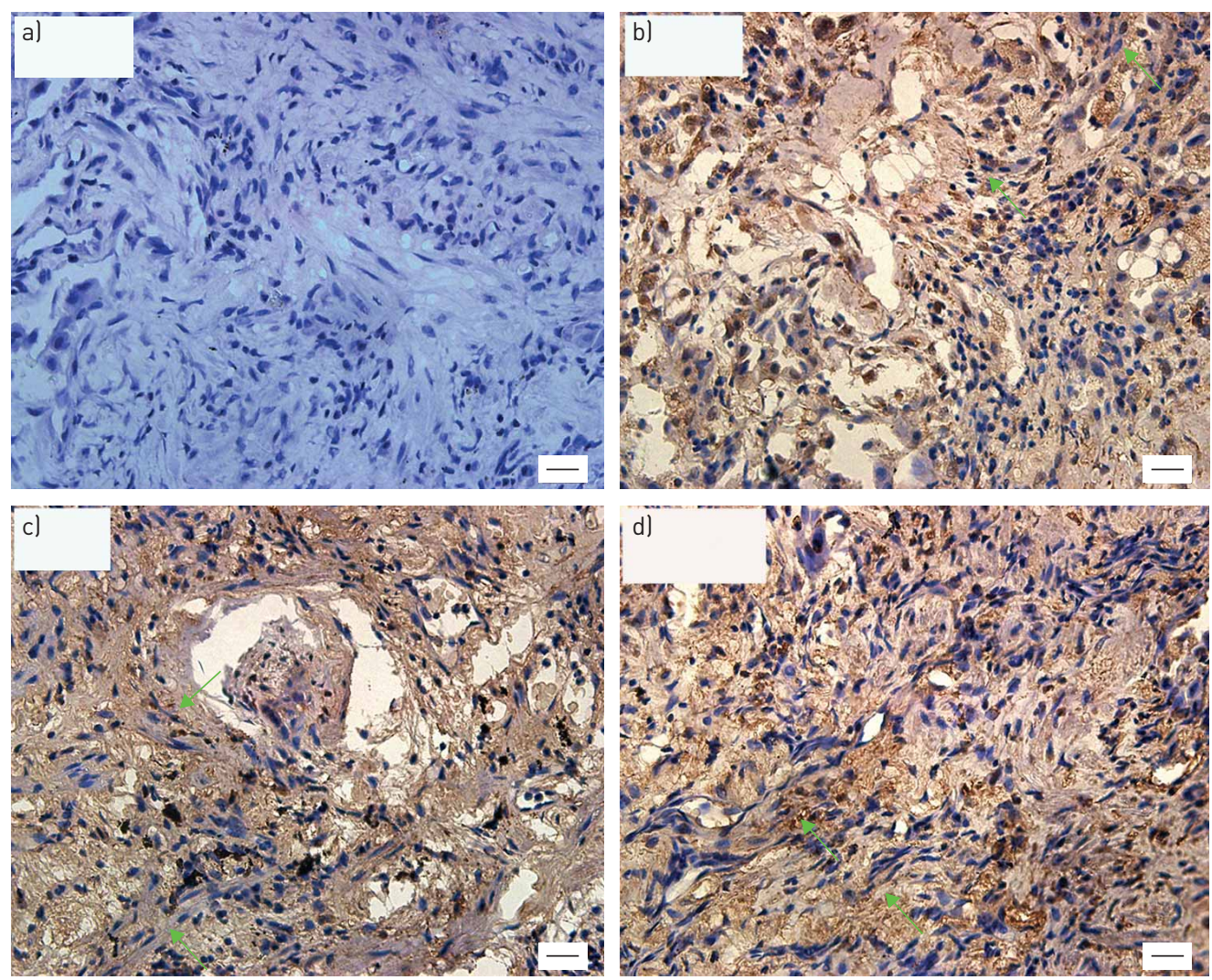

FIGURE 4 Representative images of immunohistochemical staining of discoidin domain receptor (DDR)2 in interstitial lung disease (ILD) other than idiopathic pulmonary fibrosis (IPF). The paraffin lung tissue sections from patients with non-IPF ILD were immunostained with a) mouse IgG or b-d) antibody against DDR2. The green arrows show those cells with a fibroblastic shape. Scale bars $=20 \mu \mathrm{m}$.

Established studies of the contribution of collagen-binding integrins to pulmonary fibrosis have merely centred around analysis of histological expression or of the in vitro cell behaviours, while those of the DDR family predominantly concentrated on the identification of the pathological phenotypes of genetic mouse models. Mice deficient in either $D d r 1$ or $D d r 2$ were demonstrated to be resistant to the induction of pulmonary fibrosis $[22,27]$. With regard to the mechanisms involved, DDR1 was considered primarily to regulate the function of epithelial cells and macrophages [27]. Collagen I-induced expression of DDR1 in lung fibroblasts, albeit positive, was reported to rely on the activation of DDR2 signalling [30]. The important role of DDR2 in lung fibroblasts was recently strengthened by the results from our laboratory showing that, in vitro, its expression in this type of cells could be sharply induced by TGF- $\beta$ treatment and its deficiency impedes the cell acquisition of myofibroblastic features [22]. These earlier experimental data exactly accord with our current findings that the fibroblastic cell shapes within the lung tissues of different types of ILD were strongly stained for DDR2. From the view of the type of cells they can influence, it can be conceived that DDR2 tended to be more suitable than DDR1 to be used as a therapeutic target for IPF in that myofibroblasts are major effector cells of fibrotic reaction.

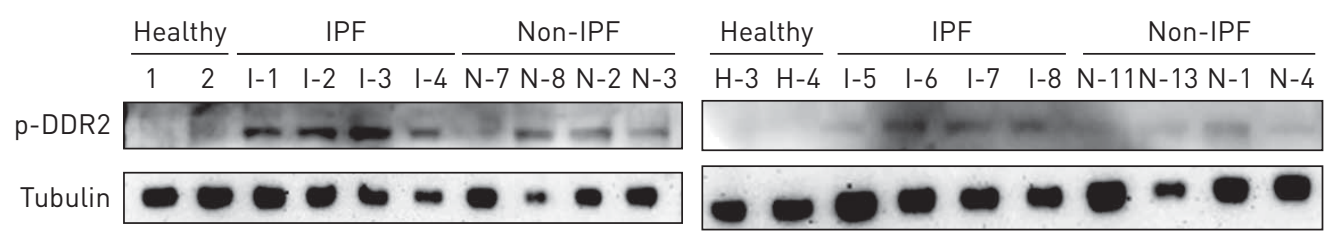

FIGURE 5 Phosphorylation levels of discoidin domain receptor (DDR)2. The lung tissue protein homogenates from the indicated interstitial lung disease (ILD) patients were separated by SDS-PAGE and then probed by Western blotting with a specific antibody against the phosphorylated form of DDR2 (p-DDR2). Idiopathic pulmonary fibrosis (IPF) patients showed the most intense level of DDR2 phosphorylation compared with healthy individuals or non-IPF ILD patients. 
DDR2 also possesses several other advantages that make it an excellent alternative molecule in designing therapeutic options against ILD. Firstly, in addition to fibrotic reaction, DDR2 can also promote the angiogenic process during pulmonary fibrosis through modulation of both endothelial cell activity and myofibroblast expression of vascular endothelial growth factor (VEGF) [22, 31]. This feature is particularly useful since nintedanib, a triple angiokinase inhibitor, has become the first approved drug for IPF treatment [32]. Secondly, we recently revealed that DDR2 not only mediates fibrillar collagen-induced signalling but is also indispensable for TGF- $\beta$-triggered activation of p38 and Akt as well as lung fibroblast conversion into myofibroblasts [22]. This function of DDR2 implies that targeting its synergistic cooperation with the TGF- $\beta$ cascade might also produce effects on the initiation of the fibrotic process. Thirdly, certain functions of DDR2, at least those requiring collagen binding and subsequent phosphorylation of DDR2, could be blocked by either neutralising antibody or kinase inhibitors. Fourthly, accumulating evidence indicates that DDR2 expression is stress inducible, which is reflected by its upregulation in response to multiple stimulation conditions such as hypoxia [26,33], TGF- $\beta$ [22] and proangiogenic cytokines [34]. This conclusion can be additionally supported by the sharp difference in DDR2 abundance between normal and IPF lungs, as disclosed by this study. The low or absent expression of DDR2 in resting cells signifies that targeting strategies against this receptor might be relatively safe.

In summary, our study reconciled the uncertainty regarding the status of the six collagen receptors in IPF, and suggests that DDR2 might be the main collagen receptor that is suitable for both diagnosis and therapy of this disease.

\section{References}

1 Johannson KA, Vittinghoff E, Lee K, et al. Acute exacerbation of idiopathic pulmonary fibrosis associated with air pollution exposure. Eur Respir J 2014; 43: 1124-1131.

2 Noble PW, Barkauskas CE, Jiang D. Pulmonary fibrosis: patterns and perpetrators. J Clin Invest 2012; 122: $2756-2762$.

3 Hutchinson J, Fogarty A, Hubbard R, et al. Global incidence and mortality of idiopathic pulmonary fibrosis: a systematic review. Eur Respir J 2015; 46: 795-806.

4 Vancheri C, Failla M, Crimi N, et al. Idiopathic pulmonary fibrosis: a disease with similarities and links to cancer biology. Eur Respir J 2010; 35: 496-504

5 Raghu G, Selman M. Nintedanib and pirfenidone. New antifibrotic treatments indicated for idiopathic pulmonary fibrosis offer hopes and raises questions. Am J Respir Crit Care Med 2015; 191: 252-254.

6 Collard HR. Where do we go from here? Clinical drug development in idiopathic pulmonary fibrosis. Eur Respir J 2015; 45: 1218-1220.

7 Canestaro WJ, Forrester SH, Raghu G, et al. Drug treatment of idiopathic pulmonary fibrosis: systematic review and network meta-analysis. Chest 2016; 149: 756-766.

8 Wilson MS, Wynn TA. Pulmonary fibrosis: pathogenesis, etiology and regulation. Mucosal Immunol 2009; 2: 103-121.

$9 \quad$ Wynn TA. Integrating mechanisms of pulmonary fibrosis. J Exp Med 2011; 208: 1339-1350.

10 Woodcock HV, Maher TM. The treatment of idiopathic pulmonary fibrosis. F1000Prime Rep 2014; 6: 16.

11 Raghu G, Scholand MB, de Andrade J, et al. FG-3019 anti-connective tissue growth factor monoclonal antibody: results of an open-label clinical trial in idiopathic pulmonary fibrosis. Eur Respir J 2016; 47: 1481-1491.

12 Kolb MRJ, Gauldie J. Idiopathic pulmonary fibrosis: the matrix is the message. Am J Respir Crit Care Med 2011; 184: 627-629.

13 Parker MW, Rossi D, Peterson M, et al. Fibrotic extracellular matrix activates a profibrotic positive feedback loop. J Clin Invest 2014; 124: 1622-1635.

14 Booth AJ, Hadley R, Cornett AM, et al. Acellular normal and fibrotic human lung matrices as a culture system for in vitro investigation. Am J Respir Crit Care Med 2012; 186: 866-876.

15 Thannickal VJ, Henke CA, Horowitz JC, et al. Matrix biology of idiopathic pulmonary fibrosis: a workshop report of the national heart, lung, and blood institute. Am J Pathol 2014; 184: 1643-1651.

16 Rittie L. Another dimension to the importance of the extracellular matrix in fibrosis. J Cell Commun Signal 2015; 9: 99-100.

17 Leitinger B, Hohenester E. Mammalian collagen receptors. Matrix Biol 2007; 26: 146-155.

18 Popova SN, Lundgren-Akerlund E, Wiig H, et al. Physiology and pathology of collagen receptors. Acta Physiol (Oxf) 2007; 190: 179-187.

19 Leitinger B. Discoidin domain receptor functions in physiological and pathological conditions. Int Rev Cell Mol Biol 2014; 310: 39-87.

20 Vogel W, Gish GD, Alves F, et al. The discoidin domain receptor tyrosine kinases are activated by collagen. Mol Cell 1997; 1: 13-23.

21 Herrera-Herrera ML, Quezada-Calvillo R. DDR2 plays a role in fibroblast migration independent of adhesion ligand and collagen activated DDR2 tyrosine kinase. Biochem Biophys Res Commun 2012; 429: 39-44.

22 Zhao $\mathrm{H}, \mathrm{Bian} \mathrm{H}, \mathrm{Bu} \mathrm{X}$, et al. Targeting of discoidin domain receptor 2 (DDR2) prevents myofibroblast activation and neovessel formation during pulmonary fibrosis. Mol Ther 2016; 24: 1734-1744.

23 Luo BH, Carman CV, Springer TA. Structural basis of integrin regulation and signaling. Annu Rev Immunol 2007; 25: 619-647.

24 Barczyk M, Carracedo S, Gullberg D. Integrins. Cell Tissue Res 2010; 339: 269-280.

25 Xia H, Seeman J, Hong J, et al. Low $\alpha_{2} \beta_{1}$ integrin function enhances the proliferation of fibroblasts from patients with idiopathic pulmonary fibrosis by activation of the $\beta$-catenin pathway. Am J Pathol 2012; 181: 222-233.

26 Yoshida K, Park A, Ozakil S, et al. Interaction of calcium- and integrin-binding protein 1 with integrin $\alpha 11$ and its possible involvement in pulmonary fibrosis. Adv Biol Chem 2014; 4: 59-66. 
27 Avivi-Green C, Singal M, Vogel WF. Discoidin domain receptor 1-deficient mice are resistant to bleomycin-induced lung fibrosis. Am J Respir Crit Care Med 2006; 174: 420-427.

28 Honda E, Yoshida K, Munakata H. Transforming growth factor-beta upregulates the expression of integrin and related proteins in MRC-5 human myofibroblasts. Tohoku J Exp Med 2010; 220: 319-327.

29 Zeltz C, Lu N, Gullberg D. Integrin $\alpha 11 \beta 1$ : a major collagen receptor on fibroblastic cells. Adv Exp Med Biol 2014; 819: 73-83.

30 Ruiz PA, Jarai G. Collagen I induces discoidin domain receptor (DDR) 1 expression through DDR2 and a JAK2-ERK1/2-mediated mechanism in primary human lung fibroblasts. J Biol Chem 2011; 286: 12912-12923.

31 Zhang S, Bu X, Zhao H, et al. A host deficiency of discoidin domain receptor 2 (DDR2) inhibits both tumour angiogenesis and metastasis. J Pathol 2014; 232: 436-448.

32 McCormack PL. Nintedanib: first global approval. Drugs 2015; 75: 129-139.

33 Ren T, Zhang W, Liu X, et al. Discoidin domain receptor 2 (DDR2) promotes breast cancer cell metastasis and the mechanism implicates epithelial-mesenchymal transition programme under hypoxia. J Pathol 2014; 234 : 526-537.

34 Katanasaka Y, Asai T, Naitou H, et al. Proteomic characterization of angiogenic endothelial cells stimulated with cancer cell-conditioned medium. Biol Pharm Bull 2007; 30: 2300-2307. 